\title{
Towards optimal snapshot location for POD model order reduction in optimal control
}

Alessandro Alla, Carmen Gräßle, Michael Hinze

\{alessandro.alla, carmen.graessle, michael.hinze\}@uni-hamburg.de

\section{Introduction}

We present the approximation of an optimal control problem for linear parabolic PDEs. The method is based on a model reduction technique using Proper Orthogonal Decomposition (POD-MOR). POD-MOR is a Galerkin approach where the basis functions are obtained upon information contained in time snapshots of the parabolic PDE related to given input data. We show that it is important to have knowledge about the controlled system at the right time instances. For the determination of the time instances (snapshot locations) we propose an a-posteriori error control which is based on a reformulation of the optimality system as a second order in time and fourth order in space elliptic system which is approximated by a space-time finite element method. Finally, we present numerical tests to illustrate our approach and to show the effectiveness of the method in comparison to existing approaches.

\section{Problem Formulation}

We deal with a standard linear quadratic problem where the cost functional we want to minimize is

$$
\min _{(y, u) \in \mathcal{Y} \times \mathcal{U}} J(y, u):=\frac{1}{2}\left\|y-y_{d}\right\|_{L^{2}\left(\Omega_{T}\right)}^{2}+\frac{\alpha}{2}\|u\|_{L^{2}\left(\Omega_{T}\right)}^{2}
$$

subject to the following constraint

$$
\left\{\begin{aligned}
y_{t}(x, t)-\Delta y(x, t) & =u(x, t)+f(x, t) & & \text { in } \Omega \times(0, T] \\
y(x, t) & =0 & & \text { on } \partial \Omega \times(0, T] \\
y(x, 0) & =y_{0}(x) & & \text { in } \Omega
\end{aligned}\right.
$$

where $\Omega \subset \mathbb{R}^{n}$ is an open bounded domain with smooth boundary, $y_{0} \in H_{0}^{1}(\Omega), \alpha, T>0$, $\mathcal{Y}:=W(0, T), \mathcal{U}:=L^{2}\left(\Omega_{T}\right)$ and $y_{d}, f \in L^{2}\left(\Omega_{T}\right)$

Optimality system:

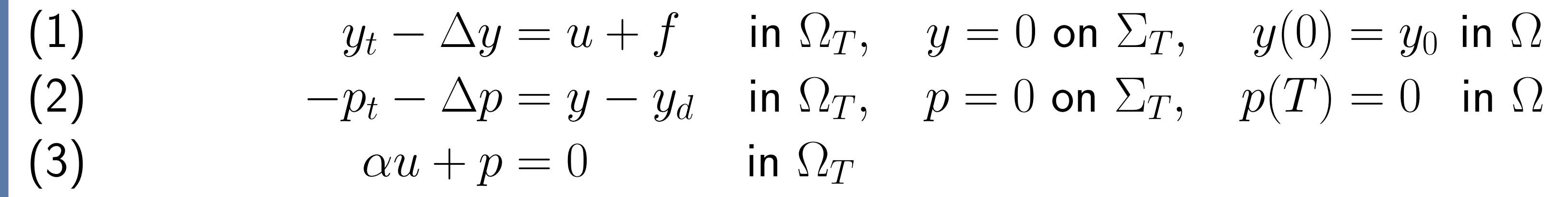

\section{Model Order Reduction}

\section{Proper Orthogonal Decomposition}

Given is a snapshot set $\mathcal{V}:=\operatorname{span}\left\{y\left(t_{1}\right), \ldots, y\left(t_{m}\right)\right\} \subset \mathbb{R}^{n}$

We look for an orthonormal basis $\left\{\psi_{i}\right\}_{i=1}^{\ell}$ in $\mathbb{R}^{n}$ with $\ell \in\{1, \ldots, d\}, d:=\operatorname{dim} \mathcal{V} \leq n$ such that

$$
J\left(\psi_{1}, \ldots, \psi_{\ell}\right)=\sum_{j=1}^{m} \beta_{j}\left\|y\left(t_{j}\right)-\sum_{i=1}^{\ell}\left\langle y\left(t_{j}\right), \psi_{i}\right\rangle_{\mathbf{W}} \psi_{i}\right\|_{\mathbf{W}}^{2}=\sum_{i=\ell+1}^{d} \lambda_{i}
$$

reaches its minimum subject to

$$
\left\langle\psi_{j}, \psi_{i}\right\rangle_{\mathbf{W}}=\delta_{i j} \text { for } 1 \leq i, j \leq \ell,
$$

where $\beta_{j} \in \mathbb{R}^{+}, j=1, \ldots, m$. The vectors $\left\{\psi_{i}\right\}_{i=1}^{\ell}$ are called $P O D$ basis of rank $\ell$

Singular Value Decomposition: $Y=\Psi \Sigma \Phi^{T}$ is utilized for POD basis computation.

\section{Optimal Snapshot Location}

Problems:

(1) POD basis vectors $\left\{\psi_{i}\right\}_{i=1}^{\ell}$ depend on the snapshot set.

What is the quality of the POD basis for an unknown optimal control?

3) What are suitable time instances to compute snapshots?

The main idea is to generate snapshots which are input independent. We rewrite the optimality system (see[1]) as a second order in time and fourth order in space elliptic equation and we get a two point boundary value problem which does not depend on the control $u$

$$
\left\{\begin{aligned}
-y_{t t}+\Delta^{2} y+\frac{1}{\alpha} y & =\frac{1}{\alpha} y_{d} & & \text { in } \Omega_{T} \\
y & =0 & & \text { on } \Sigma_{T} \\
\Delta y & =0 & & \text { on } \Sigma_{T} \\
\left(y_{t}-\Delta y\right)(T) & =0 & & \text { in } \Omega \\
y(0) & =y_{0} & & \text { in } \Omega
\end{aligned}\right.
$$

Equation (4) is obtained by inserting equation (3) into (1) and taking the derivative with respect to time $t$. To Approximate equation (4) we follow the approach in [1] where a space-time finite element technique with a time-adaptivity scheme based on a-posteriori error estimates is performed.

Temporal residual type a-posteriori error estimation for $y$ :

$\left\|y-y_{k}\right\|_{H_{0}^{2,1}\left(\Omega_{T}\right)}^{2} \leq c \cdot \eta^{2}$
where $\eta^{2}=\sum_{j} \Delta t_{j}^{2} \int_{I_{j}}\left\|\frac{1}{\alpha} y_{d}+\frac{\partial^{2} y_{k}}{\partial t^{2}}-\Delta^{2} y_{k}-\frac{1}{\alpha} y_{k}\right\|_{L^{2}(\Omega)}^{2}+\sum_{j} \int_{I_{j}}\left\|\Delta y_{k}\right\|_{L^{2}(\Gamma)}^{2}$

where we consider the discretization in time: $0=t_{1}<t_{2}<\ldots<t_{m}=T, \Delta t_{j}=t_{j}-t_{j-1}$,

$I_{j}=\left[t_{j-1}, t_{j}\right]$

Outcome:

(1) Obtain information about a possible time grid for snapshots.

2) Obtain snapshots related to an approximation of the optimal solution.

\section{Numerical strategy}

Algorithm 1 Adaptive snapshot selection for optimal control problems.

Require: (Large) space step size $\Delta x$, number degree of freedom (dof) for the time discretization, $T>0$.

Compute a numerical approximation of (4) with temporal a-posteriori error estimation. $\rightarrow$ Obtain a time grid and an approximation of the optimal control.

Use this control to build the snapshot matrix $\mathcal{V}$.

Compute a POD basis of order $\ell$.

4: Set up and solve the reduced problem on the time adaptive grid.

\section{Numerical Test}

Chosen data: $\Omega=(0,1), T=1, \alpha=1, \bar{y}_{\text {true }}(x, t)=\sin (\pi x) \operatorname{atan}((t-1 / 2) / \varepsilon)$, $y_{d}(x, t)=\sin (\pi x)\left(\pi \cos (\pi t)-\pi^{2} \sin (\pi t)+\operatorname{atan}\left(\frac{t-1 / 2}{\varepsilon}\right)\right)$
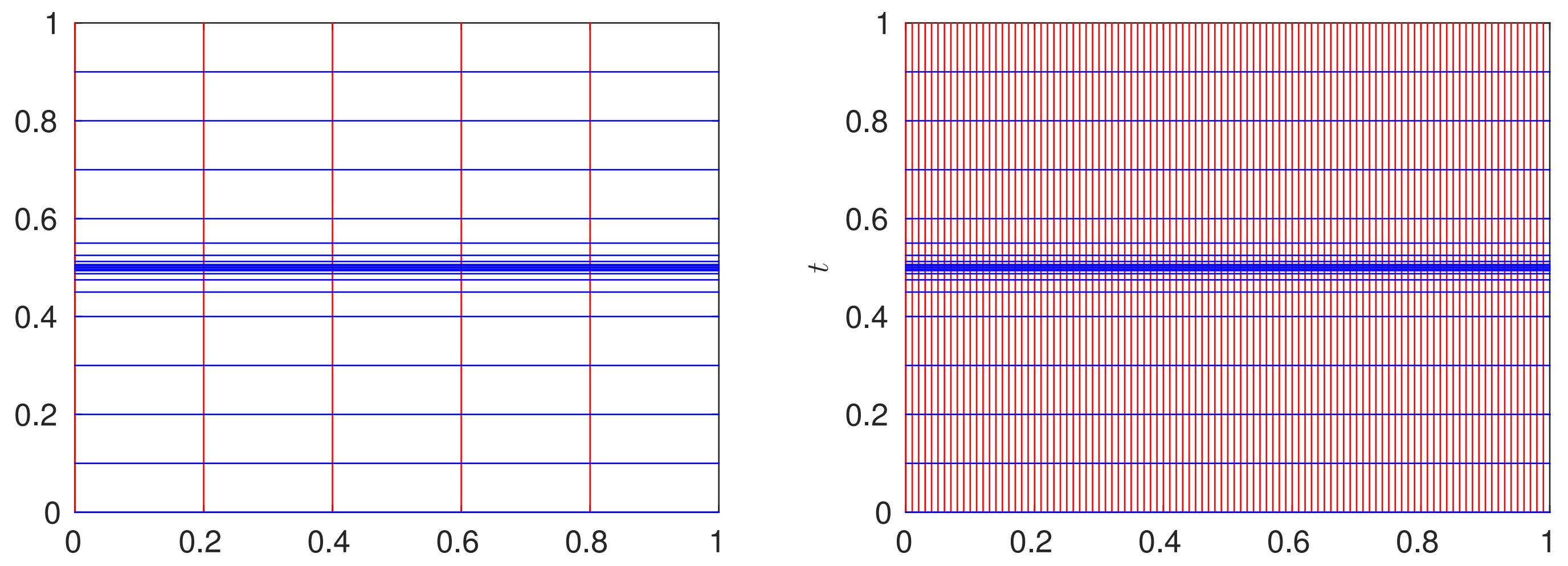

Figure 1: Adaptive space-time grids with dof $=21$ and $\Delta x=1 / 5$ (left) and $\Delta x=1 / 100$ (right) calculated by utilizing the temporal residual type a-posteriori error estimation for $y$. Obviously, temporal and spatial discretization decouple which motivates to use a large spatial discretization size in the offline computation phase in Algorithm 1 in order to achieve a fast offline stage.
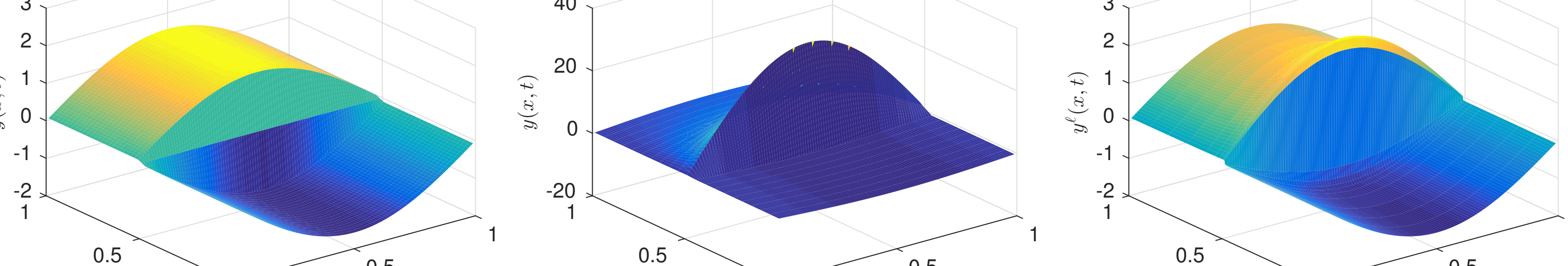

Figure 2: True optimal state $\bar{y}_{\text {true }}$ (left), approximated POD solution computed on an equidistant time grid (middle) and approximated POD solution utilizing the adaptive time grid (right) with dof $=21$.
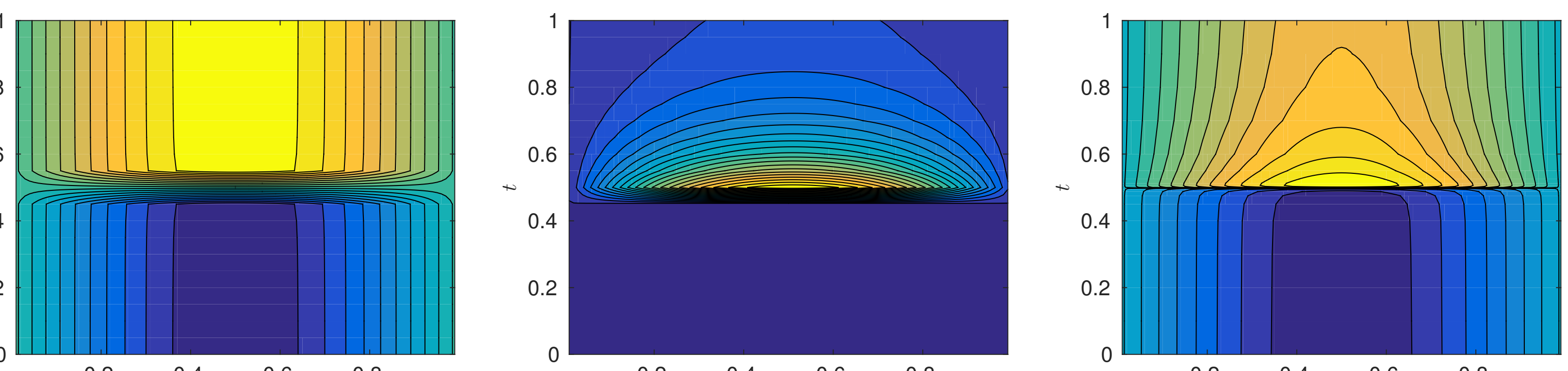

Figure 3 . Contour lines of the true optimal state $\bar{y}$ true (left), approximated POD solution computed on an equidistant time grid (middle) and approximated POD solution utilizing the adaptive time grid (right) with dof $=21$.

\begin{tabular}{c|c|c|c|c|c}
$\Delta t$ & $\left\|\bar{y}-\bar{y}^{\ell}\right\|_{L^{2}\left(\Omega_{T}\right)}$ & $\left\|\bar{u}-\bar{u}^{\ell}\right\|_{L^{2}\left(\Omega_{T}\right)}$ & $\operatorname{dof}$ & $\left\|\bar{y}-\bar{y}^{\ell}\right\|_{L^{2}\left(\Omega_{T}\right)}$ & $\left\|\bar{u}-\bar{u}^{\ell}\right\|_{L^{2}\left(\Omega_{T}\right)}$ \\
\hline $1 / 20$ & $6.7264 \cdot 10^{+00}$ & $4.7581 \cdot 10^{-01}$ & 21 & $1.8468 \cdot 10^{-01}$ & $1.1610 \cdot 10^{-02}$ \\
$1 / 46$ & $2.8634 \cdot 10^{+00}$ & $1.9863 \cdot 10^{-01}$ & 47 & $2.0049 \cdot 10^{-02}$ & $9.3412 \cdot 10^{-03}$ \\
$1 / 82$ & $1.4603 \cdot 10^{+00}$ & $1.0037 \cdot 10^{-01}$ & 83 & $1.2574 \cdot 10^{-02}$ & $5.0596 \cdot 10^{-03}$ \\
$1 / 108$ & $1.0233 \cdot 10^{+00}$ & $6.9951 \cdot 10^{-02}$ & 109 & $6.3950 \cdot 10^{-03}$ & $4.8042 \cdot 10^{-03}$
\end{tabular}

Table 1: Absolute errors between the true optimal solution and the POD suboptimal solution depending on the time discretization (equidistant: columns 1-3, adaptive: columns 4-6)
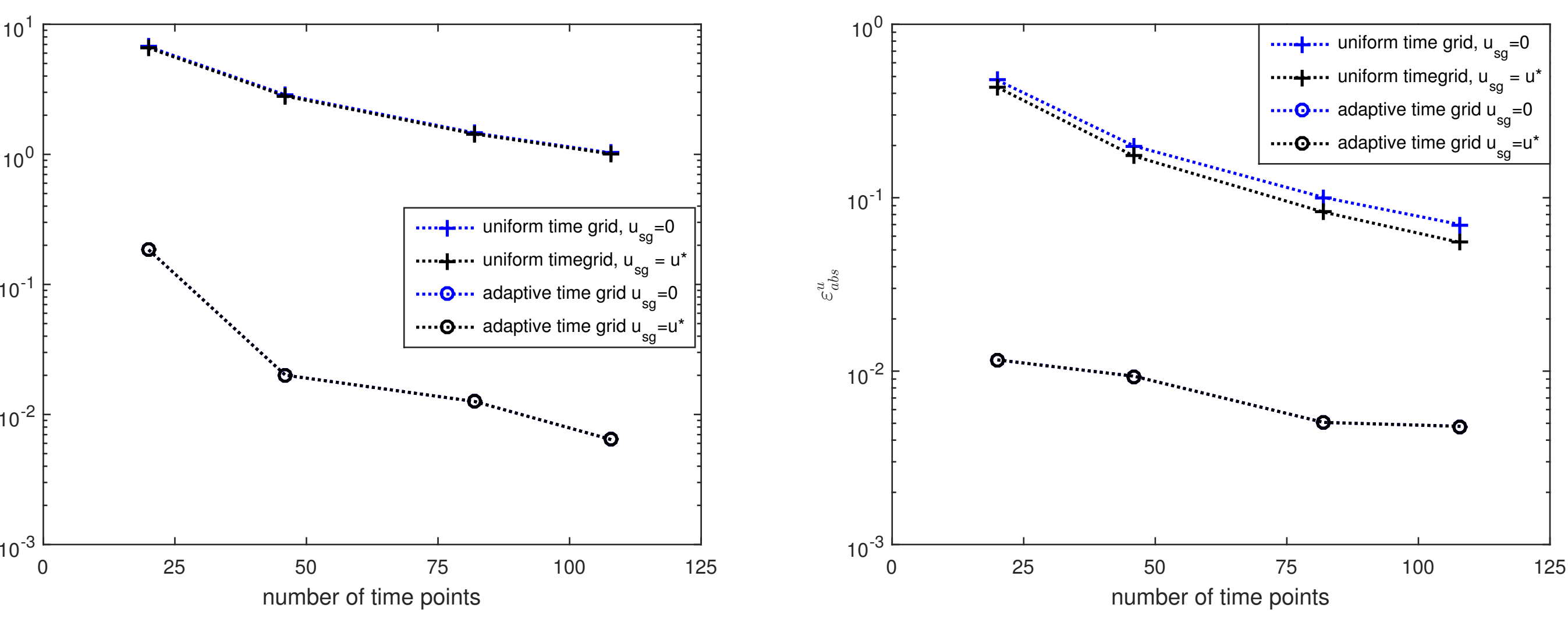

Figure 4: Effect of using different time grids on the $L^{2}$-errors between the true optimal state (control), left (right), and the POD suboptimal state (control), left (right) with optimal and uncontrolled snapshots.

\section{References}

(1) W. Gong, M. Hinze, Z.J. Zhou: Space-time finite element approximation of parabolic optimal control problems, 2013. 2 M. Gubisch, S. Volkwein. Proper Orthogonal Decomposition for Linear-Quadratic Optimal Control, 2013, 3 M. Hinze, R. Pinnau, M. Ulbrich, S Ulbrich. Optimization with PDE Constraints. Mathematical Modelling: Theory and Applications, 2009.

(1) R H.W Hoppe, Z Liv: Snapshot tocation by ert

parabolic partial differential equations, 2014

5. K. Kunisch. S. Volkwein: Proper Orthogonal Decomposition for optimality system 2008

6 K. Kunisch, S. Volkwein. Optimal Snapshot Location for computing POD basis functions, 2010.

(7) G.M. Oxberry, T. Kostova-Vassilevska, B. Arrighi and K. Chand. Limited-memory adaptive snapshot selection for proper 